\title{
The Modeling of Complex Agent Network based on BDI Agent
}

\author{
Ablimit Arxiden \\ Faculty of Computer and Information Science, Southwest University, Chongqing 400715, China \\ Faculty of Mathematics and Information, Teachers Collage of Hotan, Hotan 848000, China
}

\begin{abstract}
In this paper, on the basis of deeply analyzing the basic search algorithm, the features of complex network structure, and the complexity of the search process, multi-agent system (MAS) and complex network as two method of complex system modeling are systematically studied to draw on their features, and then a new complex agent network (CAN) model supporting complex network search process is proposed and the idea and formalization of BDI-based CAN model are defined.
\end{abstract}

\section{Keywords: CAN; MAS}

\section{INTRODUCTION}

\subsection{Kleinberg network model}

Jon Kleinberg created a social network search method based on node search. Decentralized search algorithm is applied. The study of Watts and Strogarz shows the complex networks support the decentralized search. The problem is that the results mostly depend on the rarity of the information and the diversity of network topology. When the structure of the network is too simple (i.e. structurally impoverished), a large number of relevant enormous investment is demanded to introduced, so as to search the best route from the "enquiry" to the "answer".

\subsection{Agent/MAS model based on smart technology}

Multi-Agent system (MAS) is designed to be mainly different from traditional methods: (1) static; (2) dynamic. A single Agent can be independently developed and debugged, and will not affect the use of other Agents; the system development is relatively easy and simple. These are the main advantages of establishing a mobile learning model based on multi-Agent.

\section{The idea of complex Agent network}

\subsection{The idea of complex Agent network}

To improve the intelligence of nodes, an information sharing mechanism between different nodes is established. Nodes can actively transfer information to searchers, and thus, the sharing of the information is improved in the process of search. A new smart search network model is established on the basis of retaining the original characteristics of the complex network. Through an in-depth study of the complex network search, it is found that the geographic coordinates of the established Kleinberg grid model make individual nodes fixed, but different nodes have different relations in the real network, and also each node does not fundamentally consider the effect of the search algorithm process. In the real network, the specific nodes will affect the change of the search process. 


\subsection{Analysis on the structure of complex agent network}

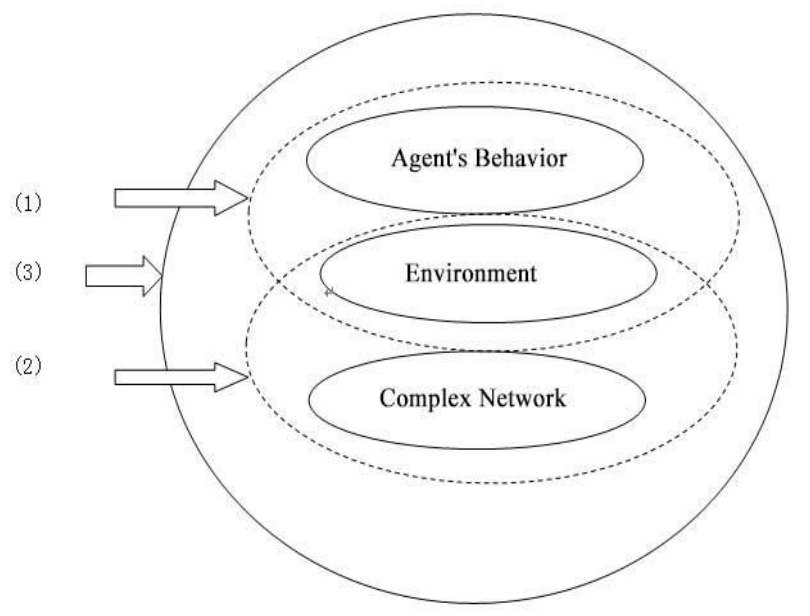

Fig.1 the modeling of Complex Agent Network

Complex Agent network (CAN) is a new network model established on the basis of multi-Agent system and the characteristics of the original complex network model, as shown in
Fig.1. The modeling of complex Agent network (CAN) can be divided into three layers that are mutually correlated and promoted, and their structural relations are as shown in Fig. 2 .

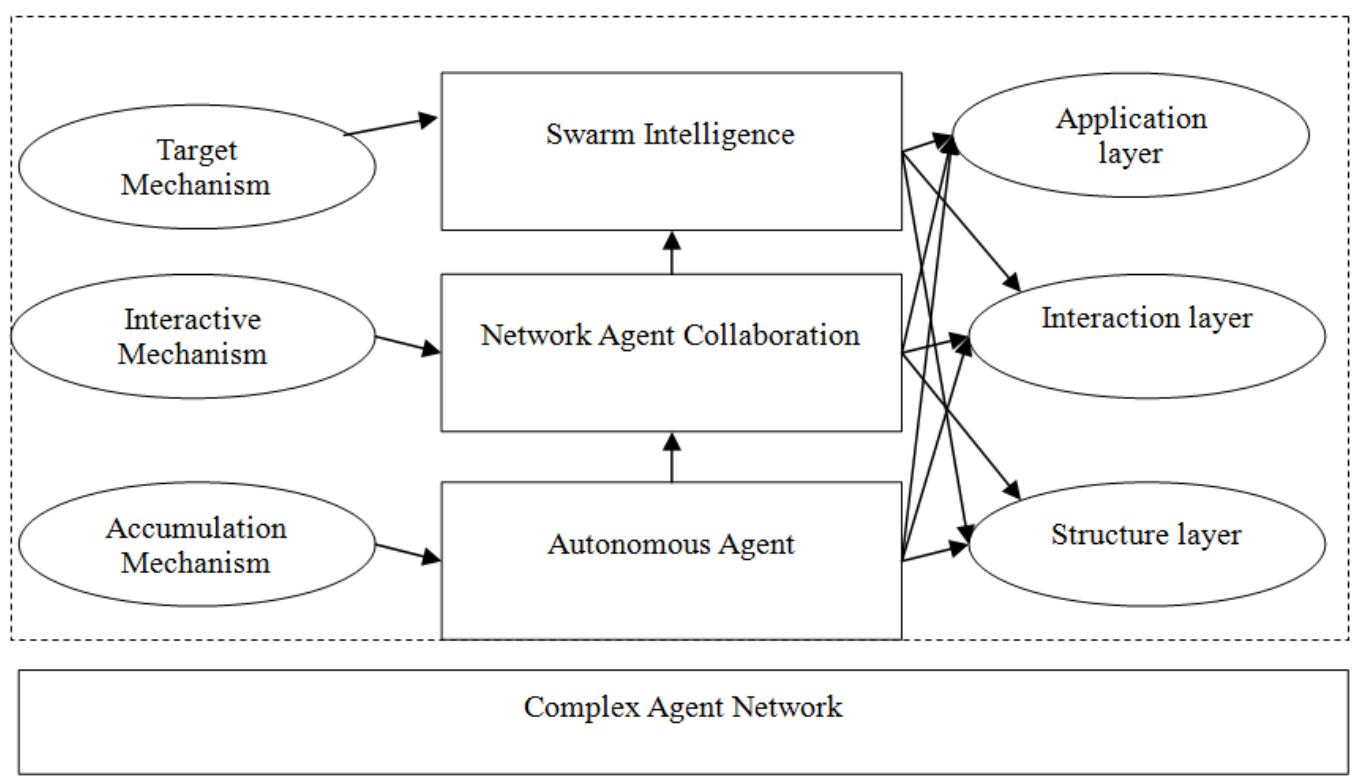

Fig. 2 the structure of Complex Agent Network

(1) The layer of structure: The layer of the structure of the model includes the specific scope needed by this study, and this is a potential requirement for establishing complex system model, choosing an appropriate abstraction layer, and determining the types and directions of the information flows in the network.

(2) The layer of interaction: It is for the individual Agent modeling in the complex 
Agent network, including the determination of each Agent's clear boundaries and the establishment of each Agent behavior.

(3) The layer of application: According to the given task objects, it completes the determination of the operation modes for the mutual behaviors between Agents.

\section{The formal description of complex Agent network}

\subsection{The formal definition of the network model}

\subsubsection{The definition of complex Agent network}

A concrete CAN network can be abstracted as a set of Agent nodes. The cooperative relationship between Agents is composed by the close degree weight function $\mathrm{W}_{\text {agents }}$ of the cooperative relationship between frontier set $E$ and Agent.

$$
\mathrm{G}_{\text {agents }}=\left(\mathrm{V}_{\text {agents }}, \mathrm{E}_{\text {agents }}, \mathrm{W}_{\text {interaction }}\right)
$$

From mathematical perspective, complex Agent network can be abstract as triples as follows:

$$
\text { CAN }(t)=\left(\mathrm{V}_{\text {agents }}(t), \mathrm{E}_{\text {agents }}(t), \mathrm{W}_{\text {interaction }}(t)\right)
$$

Where,

$N$ - the number of node Agent is $\mathrm{N}=|\mathrm{V}|$;

$M$ - the number of edge is $\mathrm{M}=|\mathrm{E}|$;

$W$ - the number of edge weight is $\mathrm{W}=|\mathrm{W}|$;

Each node of $\mathrm{V}_{\text {agents }}(t)$ is closely combined with an Agent to generate a complete whole. Each edge of $E_{\text {agents }}(t)$ is only correlated with two Agents. The edges are not only processed by the constraint of complex network topology, but also related to the internal state of the two agents. In addition, a new problem is necessary to consider: node and edge change over time. Therefore, a variable $t$ in complex Agent network is introduced for expressing the direct or indirect relationships between the change of node's state and the time. This is easy to show the dynamics of complex networks. The definition of node is extended to $\mathrm{V}_{i}\left(\operatorname{Agents}_{i}(t), t\right)$. In multi-Agent system, the same serial number $i$ is applied to agent's sequence and the node subsequence of complex network, and its own state change is driven by the internal behaviors and has a close relationship with time. From the above extended definition, it is seen that node Agent, edge, and these two nodes are affected by Agent. The above expression can be modified as follows:

$$
\begin{aligned}
& \left.\left(\mathrm{V}_{i}\left(\operatorname{Agent}_{i}(t), t\right)\right), \mathrm{Vj}\left(\operatorname{Agent}_{j}(t), t t\right)\right) \\
= & \left.\left(\mathrm{E}_{i}\left(\operatorname{Agent}_{i}(t), t\right)\right), \mathrm{E}_{\mathrm{i}, \mathrm{j}}\left(\operatorname{Agent}_{j}(t), t\right)\right)
\end{aligned}
$$

In the following, all nodes are simplified as $\mathrm{V}_{i}^{\mathrm{t}}$ (Agents $\left._{i}{ }^{\mathrm{t}}\right)$, and all edges are as $\mathrm{E}_{\mathrm{i}}^{\mathrm{t}},{ }_{\mathrm{j}}\left(\right.$ Agent $_{i}^{\mathrm{t}}$, Agent $\left._{j}^{\mathrm{t}}\right)$.

\subsubsection{The definition of interaction}

Agent acquires effective information from other agents or environment, and then modifies its own state according to the amount of the acquired information and requirements, and then sends important instructions to other Agents or environment for interactive collaboration. This is a complete process of information transfer.

3.1.3 The definitions of private information and public information

Private information refers to the information stored internally by Agent in the past. Public information refers to the information of the mutually correlated Agents, and it is local or global. Public information includes the heuristic information about some specific problems, but also the knowledge and experience accumulated by the so-called Agent in the initial stage of the searching process.

\subsection{The definition of Agent model}

In this paper, how Agent makes reasonable and effective reasoning under the conditions of external environment and internal environment changes is analyzed using BDI Agent as an example. The types of Agents in section 1 and section 2 are used as main references, and then the definitions and descriptions of the structure, relationship, interaction, communication and behavior of Agent are further studied. The behaviors in all characteristics of Agent can 
affect the structural characteristics of Agent, and also the problems such as relationships, interactions, and communications are solved, and therefore, the description of Agent's behaviors in Agent's description is especially critical. Then, Agent's behavior description can be divided into automata, state diagram, and formal logic, and so on. The formal logic is applied to describe the behavior of Agent here.

After the above analysis, formalized state $S$ behavior $B$ can constitute Agent, and its expression is as follows:

$$
\text { Agent }=\left\langle S_{t}, B\right\rangle
$$

\subsubsection{The expression of state}

In the CAN model, simulation demonstration and program tracking the evolution of an object system is to make simulation, and it is a form of expression for all attributes of an abstract thing. That is, the activities, status and stage of the studied object are statically described at a specified time, and the independent state defines the values of all attributes of a thing.

(1) Definition 1 (event): The change of the system state at a particular time is called as Event $(e)$.

(2) Definition 2 (state): The expressional form for all properties of an abstract thing is called as Status. For the state of the object at a specific time, it statically describes a phase. $S^{t}$ is the state of the system at a specified time $(t)$.

$S=\left(S_{1}, S_{2}, S_{3}, S_{4}, \ldots \ldots, S_{n}\right)^{T}$

(3) Definition 3 (the domain of state): The domain of state is used for describing the attributes of some features of Agent in the model. $S_{1}, S_{2}, S_{3}, S_{4}, \ldots \ldots, S_{n}$ in $S=\left(S_{1}, S_{2}, S_{3}\right.$, $\left.S_{4}, \ldots \ldots, S_{n}\right)^{T}$ is the domain of state.

(4) Definition 4 (the space of state): If a system has $N$ states, these state domains or domain values constitute the $\mathrm{N}$-dimensional state space, as follows:

$S 1=\left(d_{1}, d_{2}, d_{3}, d_{4}, \ldots \ldots, d_{n}\right) T$
Where, the variables of the domain $\left(d_{1}, d_{2}, d_{3}\right.$, $d_{4}$,) of state are expressed with $d_{n}$.

(5) Definition 5 (transfer function): $Q: S \rightarrow S$ is a mapping of the system, namely, the transfer function.

The state space of the system is expressed with $S$. The program for the state change of the system is the system state space. It is assumed there are $N$ domains of state in the system, and an $N$-variable equation can be expressed as the transfer function: $Q=f\left(S_{0}\right)=f\left(d_{1}, \quad d_{2}, \quad d_{3}\right.$, $\left.d_{4}, \ldots \ldots, d_{n}\right)$. In the following, the state space will be further introduced. The following rule is a description of the state domain for the state domain of a system: uncertainty rule and complete rule. Thus, the state of Agent can be expressed through the first-order predicate formula. A predicate atomic formula is the definition of a state domain of Agent, as follows:

Predicates (item 1, [item 2], [it

em 3], $\cdots \cdot$, [item $n$ ]

The functional vocabularies such as constant, number, and name are items in the above. The predicate of Agent quantity is expressed with Agent Count. For example, Agent Count (5): It can express the state domain of Agent quantity, and 5 is the current state value. In addition, it can express relationship and properties, etc. It is necessary to note that inside and outside states are the two states of Agent.

\subsubsection{The expression of behavior}

The related concepts of Agent are defined and introduced in the following.

Definition 1 (sequence): Sequence is an orderly arrangement mode for all elements of a set. The definitions of the three logical operators are as follows: $p$ and $q$ as two elements are assumed, and the logical operator " $p \rightarrow q$ " is defined: " $p \vee$ $q$ " means $p$ and $q$ are paralleling; " $p \wedge q$ " means " $p$ or $q$ ".

Definition 2 (action): Changing Agent or its external environmental state is called as Action. It executes the smallest unit, while the smallest 
units constitute the activities. It is expressed with Act.

All actions can be expressed as triples:

$A c t=\left\langle S_{s}, O p, S_{e}\right\rangle$

$\left.<S_{s}:\left\{d_{1}, d_{2}, \ldots \ldots, d_{n}\right\} T\right\rangle$

$\left\langle O p: f\left(d_{1}, d_{2}, \ldots \ldots, d_{n}\right)\right\rangle$

$<S_{e}:\left\{d_{1}, d_{2}, \ldots \ldots, d_{n}\right\} T>$

Where,

$S_{e}$ - the state of termination;

$S_{s}$ - the initial state;

$O p$-the action function and the state transfer function $O p$;

All types of operation description languages can be expressed with transfer function, and even can be described with editing language. Furthermore, multiple actions can be expressed as follows according to the action sequence.

$S A=\langle A c t s, Q\rangle$

Acts is obtained by the limited actions of set, while the subset of all actions of Agent is Acts; the formula for the combination of the orderly arrangements of all actions can be expressed with $Q$. The following formula can be used for expressing a sequence of actions.

$$
S A_{1}=\left\langle\operatorname{Acts}_{1}, Q_{1}\right\rangle
$$

Where,

$$
\begin{aligned}
& A c t_{1}=\left\langle A c t_{1}, A c t_{2}, A c t_{3}, A c t_{4}\right\rangle \\
& Q_{1}=\left\langle\left(A c t_{1} \wedge A c t_{2}\right) \rightarrow\left(A c t_{3} \vee A c t_{4}\right)\right\rangle
\end{aligned}
$$
$A^{*}$.

The sequence of all actions is expressed with

Definition 3 (Activity): The orderly startup composed of multiple spontaneous actions of inspired event or Agent is called as Activity (Atv). The expression for the activity is shown as follows:

$A t v=\langle T, E, A c t s>$
$T$ is the set of the targets of activity. The description for the targets of activity is contained in activity. $E$ is the set of events and can describe environmental stimulus Agent, and it is expressed with an empty set. This shows that the target of the activity is inspired by the subset of the actions of Agent. Acts is an expression for the sequence of actions, namely, Acts $\in A^{*}$. The expression for the activity is shown as follows:

$$
\begin{aligned}
& A t v_{1}=\left\langle T_{1}, E_{1}, A c t s_{1}\right\rangle \\
& T_{1}=\{\text { Target Description }\} \\
& E_{1}=\langle e 1, \text { Event Description }\rangle \\
& \operatorname{Acts}_{1}=\langle A c t s, Q\rangle \\
& A c t s_{1}=\left\langle A c t_{1}, A c t_{2}, A c t_{3}, A c t_{4}\right\rangle \\
& Q_{1}=\left\langle\left(A c t_{1} \wedge A c t_{2}\right) \rightarrow\left(A c t_{3} \vee A c t_{4}\right)\right\rangle
\end{aligned}
$$

What do these formulas mean? An activity of Agent is an inspiration by time or a spontaneous startup made by a series of targeted activities. Internal activity is inspired, while external activities are started up spontaneously.

Defining 4 (Behavior): The characteristics of abstract Agent are called as behaviors $(B)$. It gathers all internal and external activities of Agent.

$$
B=\left\langle T^{*}, E^{*}, A c t^{*}, A v t^{*}\right\rangle
$$

Where,

$T^{*}$ - the table of the sets of all actions of Agent;

$E^{*}$ - the set of all events perceived by Agent;

Act ${ }^{*}$ - the set of all internal actions of Agent;

$A v t^{*}$ - the set of all external actions of Agent;

\section{Conclusion}

In this paper, a new complex Agent network method supporting the complex network search process is proposed by effectively absorbing the characteristics of both MAS and CAN, and then the ideas and formal definitions of CAN model 
are presented. In this method, the related state and behavior of Agent are described using individual Agent model, and also the complexity of the collaboration between the real complex network nodes is described using the virtual complex Agent network (CAN), for the main purpose of better describing the related problems of the complex network dynamics behaviors and the complexity between individual layer and group layer.

\section{References}

[1] Karlsson D, Jansson A, Normark B H, et al. An Individual-based Network Model to Evaluate Interventions for Controlling Pneumococcal Transmission [J]. BMC Infectious Diseases. 2008, 8.

[2] Vickers D M, Osgood N D. A Unified Framework of Immunological and Epidemio-logical Dynamics for the Spread of Viral Infections in a Simple Network-based Population [J]. Theoretical Biology and Medical Modelling. 2007, 4. 